\title{
On the Analysis of Scope Ambiguities in Comparative Constructions: Converging Evidence from Real-Time Sentence Processing and Offline Data *
}

\author{
Micha Y. Breakstone \\ HUJI and MIT \\ Danny Fox \\ HUJI and MIT
}

\author{
Alexandre Cremers \\ SIGMA - ENS \\ Martin Hackl \\ MIT
}

\begin{abstract}
This paper compares two accounts of an ambiguity that arises when a comparative phrase containing an exactly differential is embedded under an intensional operator (Heim 2000). Under one account, the comparative phrase is responsible for the ambiguity (the $e r$-scope theory), and, under the other, the ambiguity is attributed to the exactly phrase (the exactly-scope theory). We present converging evidence from the distribution of de re and de dicto readings and real time sentence processing that supports the $e r$-scope theory. Since the $e r$-scope theory presupposes a quantificational analysis of the comparative, such an analysis is ipso facto supported by our results.
\end{abstract}

Keywords: Comparatives, Scope, Sentence Processing

\section{Introduction}

The syntax and semantics of comparatives have been a source of much debate in linguistic theory. Central to this debate is the question of whether comparatives should be analyzed as quantifiers over degrees (see, e.g., von Stechow 1984 vs. Kennedy 1997). In support of a quantificational analysis, Heim 2000 presents certain ambiguities which can arise when sentences containing a comparative phrase are embedded under intensional operators. Heim analyzes these as scope ambiguities resulting from the ability of the comparative phrase to be interpreted either above or below the intensional operator. However, Oda 2008 and Beck 2009, henceforth

* We thank the audience at SALT 21, Irene Heim, Toshiko Oda, Sigrid Beck, Philippe Schlenker, Benjamin Rozenfeld and the audience of MIT course 24.979 in the Spring of 2010 for helpful comments and suggestions. Also, this paper was made possible in part by the Euryi grant "Presupposition: a formal pragmatic approach", given by the European Science Foundation, as well as the President's Scholarship Program at HUJI. 
O\&B, present an account of some of Heim's data which is consistent with a nonquantificational analysis of the comparative phrase. In this paper we spell out a prediction that could distinguish Heim's interpretation of the facts from O\&B's. We then present empirical evidence that favors Heim's interpretation, adding further support to a quantificational analysis for the comparative.

The ambiguity that we will focus on is illustrated in (1), modeled after Heim 2000, which contains both the comparative morpheme er and the differential phrase exactly 2 inches.

(1) Mary is 6 feet tall. John is required to be exactly 2 inches taller than that.

Under one interpretation, henceforth the "exactly reading", what is required is that John's height be exactly $6^{\prime} 2^{\prime \prime}$. Under the second interpretation, there could be a range of heights that would satisfy the requirements: they could be met as long as John's height is greater or equal to $6^{\prime} 2^{\prime \prime}$ - the "at least reading". To see that the at least reading exists, imagine that when the sentence is uttered it is presupposed that there is only a minimal height requirement. Under such circumstances, the sentence could be understood as specifying the minimal requirement.

Both Heim and O\&B analyze the ambiguity as a scope ambiguity, with the exactly reading resulting from a structure in which a quantifier over degrees has the narrowest scope possible and the at least reading resulting from a structure in which it takes scope over the modal (e.g., moved there by Quantifier Raising, QR). We will, thus, sometimes refer to the exactly reading as a "surface scope reading" and to the at least reading as an "inverted scope reading". 1 The debate between the two proposals centers on the identity of the quantifier that undergoes long-distance QR. For Heim it is the comparative quantifier headed by -er - the phrase exactly 2 inches -er than that - and for O\&B it is simply the differential phrase exactly 2 inches.

The choice between the two proposals has an obvious bearing on the syntax and semantics of the comparative. Heim's proposal (henceforth, the "er-scope theory") presupposes an analysis of the comparative as a quantifier over degrees. O\&B's proposal (henceforth, the "exactly-scope theory") is compatible with a nonquantificational analysis of the comparative (e.g. that of Kennedy 1997).

We propose to distinguish the two proposals on the basis of predictions pertaining to the position of the than-clause under the at least (inverted scope) interpretation. We will see that by the er-scope theory, the than-clause cannot be in the scope of the intensional operator for this interpretation to be available whereas under the exactly-scope theory, no such restriction is predicted. We present two kinds of evidence that the restriction holds: (i) off-line data based on correlations between scope and an independent diagnostic for the position of the than-clause; and (ii)

1 The terms "surface scope" and "inverted scope" are more general, as we will see, and cover cases where the modal operator has existential force resulting in an "at most" reading under inverse scope. 
data from real time sentence processing suggesting that upstream commitment to the inverted scope reading entails downstream commitment to a high position of the than-clause. The convergence of evidence will support the er-scope theory. It will, also, yield further support for the assumptions about language processing that enter into the interpretation of the real-time data.

\section{Two Theories: er-scope versus exactly-scope}

\subsection{Heim's er-scope Proposal}

Based on the existence of the at least reading, and on other evidence presented in the literature, we will follow Heim in assuming an at least interpretation for gradable adjectives, as in (2).

$$
\llbracket \operatorname{tall} \rrbracket^{w}=\lambda d_{d} \cdot \lambda x_{e} \cdot \operatorname{Height}_{w}(x) \geq d
$$

If the degree argument of the adjective is saturated by a variable and the individual argument is saturated by an individual denoting expression, we will arrive at a denotation such as that in (3) (once the variable is abstracted over):

$$
\llbracket \text { John is } d \text {-tall } \rrbracket^{w}=\lambda d \text {.Height }{ }_{w}(j) \geq d
$$

For ease of presentation, let's look at the variant of (1) given below, with the pronominal that replaced with 6 feet (a name for the degree it denotes).

(1') John is required to be exactly 2 inches taller than 6 feet.

To derive the two readings, Heim assumes that the complex constituent exactly 2 inches -er than 6 feet can move by QR to two different scope positions, yielding the two structures in (4).

a. required [[exactly $2^{\prime \prime}$-er than Mary $]_{1}$ John be $t_{1}$-tall $]$

b. [exactly $2^{\prime \prime}$-er than Mary $]_{1}\left[\right.$ required [John be $t_{1}$-tall]]

The structure in (4a), in which the comparative phrase moves locally, receives the exactly interpretation, and the structure in (4b), in which it moves non-locally, receives the at least interpretation. This semantic result follows under the assumption that the comparative phrase is a quantifier over degrees with the denotation in (5), as we can see in the calculation in (6).

$$
\llbracket \operatorname{exactly} 2^{\prime \prime} \text {-er than Mary } \rrbracket=\lambda P_{d t} .\left[\operatorname{Height}(M)+2^{\prime \prime}=\max (P)\right]
$$


In the calculation we highlight a part of the analysis which still needs to be fleshed out, namely an explanation of the way the denotation of the comparative phrase is derived from its component parts. The missing piece, which ends up rather central to our discussion, seems to be an account of the meaning of the node $\alpha$ (see $\S 2.4$ below).

a. exactly reading for Heim

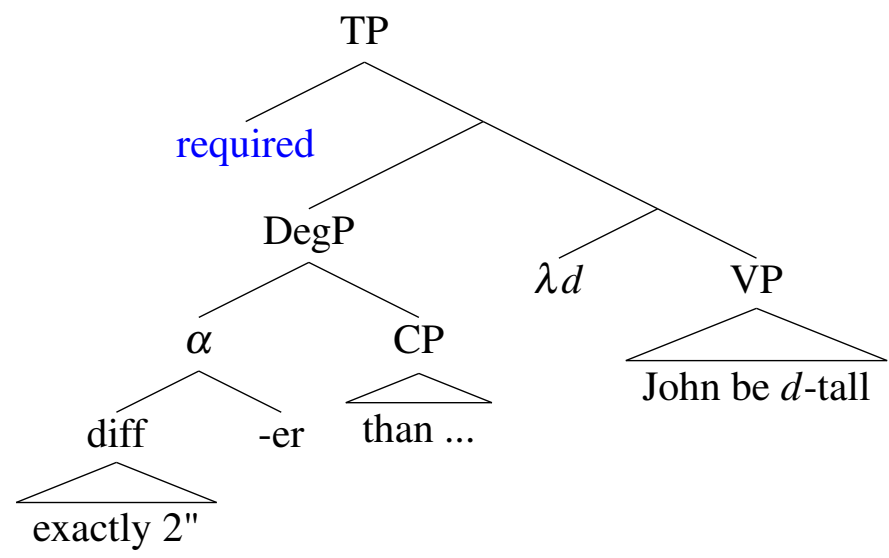

$\llbracket \alpha \rrbracket=\lambda P_{d t} \cdot \lambda Q_{d t} \cdot[\max P+2 "=\max Q]$

$\llbracket \mathrm{CP} \rrbracket=\lambda d \cdot d=6$ feet

$\llbracket \operatorname{DegP} \rrbracket=\lambda Q_{d t} \cdot[6$ feet $+2 "=\max Q]$

$\llbracket \mathrm{TP} \rrbracket^{w_{0}}=1$ iff $\forall w\left[w \in A c c_{w_{0}} \Rightarrow 6\right.$ feet $+2 "=\max \{\lambda d . \mathrm{J}$ is $d$-tall in $\left.w\}\right]$

b. at least reading for Heim

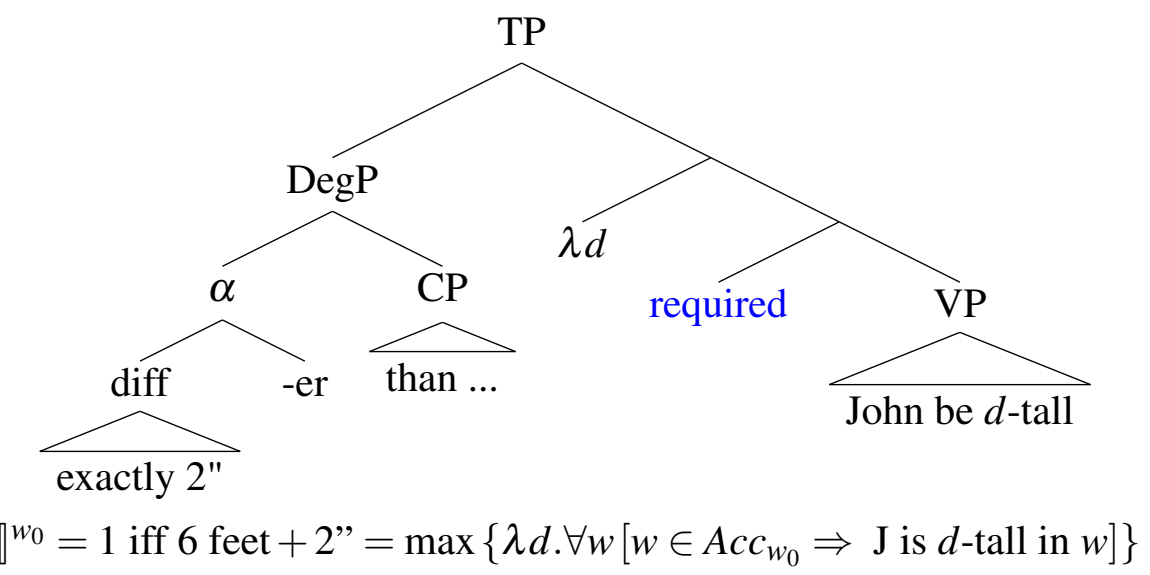




\section{2 exactly-scope Theory}

Beck 2009, crediting Oda 2008, argues that the ambiguity in (1) should be attributed to the scope of the differential phrase, exactly $2^{\prime \prime}$, which she suggests is itself a quantifier over degrees, (7).

$$
\llbracket \operatorname{exactly} 2^{\prime \prime} \rrbracket=\lambda P_{d t} \cdot\left[\max (P)=2^{\prime \prime}\right]
$$

This quantifier can move to two different positions and it is this movement that is responsible for the ambiguity, as we will see in (10). When exactly 2 inches stays below the modal, as in (8a), we get the exactly interpretation. And when it moves above the modal we get the at least interpretation. Crucially, for our purposes, this inverted scope reading does not depend on inverse scope for the comparative phrase. We derive the same interpretation whether or not this phrase moves above the modal (i.e., under the two variants in (8b)).
a. required $\left[\left[\text { exactly } 2^{\prime \prime}\right]_{2}\left[\left[t_{2} \text {-er than } 6^{\prime}\right]_{1}\left[\right.\right.\right.$ John be $t_{1}$ tall $\left.\left.]\right]\right]$
b. i. [exactly $\left.2^{\prime \prime}\right]_{2}\left[\left[t_{2} \text {-er than } 6^{\prime}\right]_{1}\left[\right.\right.$ required [John be $t_{1}$-tall $\left.\left.]\right]\right]$ ii. [exactly $\left.2^{\prime \prime}\right]_{2}\left[\right.$ required $\left[\left[t_{2} \text {-er than } 6^{\prime}\right]_{1}\left[\right.\right.$ John be $t_{1}$-tall $\left.\left.]\right]\right]$

Whether or not the comparative is quantificational is not important for the result, because, even if it is quantificational, its scope relative to the modal has no detectable consequences for the truth conditions (see Heim 2000). For concreteness, we employ the quantificational analysis in (9).

$$
\llbracket \text {-er } \rrbracket=\lambda d_{d} \cdot \lambda P_{d t} \cdot \lambda Q_{d t} \cdot[\max (Q) \geq \max (P)+d]
$$

(10) a. exactly reading for Oda/Beck (differential takes narrow scope)

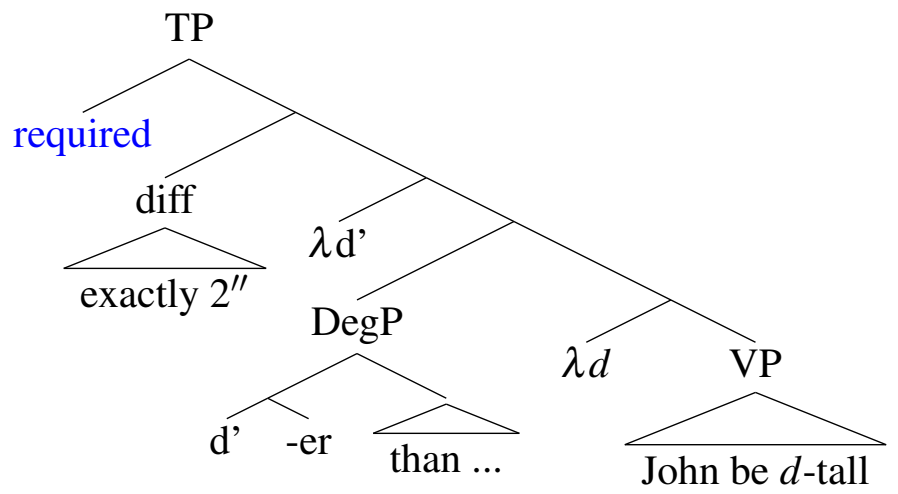

$\llbracket \operatorname{exactly} 2^{\prime \prime} \rrbracket=\lambda P_{d t} \cdot\left[\max (P)=2^{\prime \prime}\right]$

$\llbracket$-er $\rrbracket=\lambda d_{d} \cdot \lambda P_{d t} \cdot \lambda Q_{d t} \cdot[\max (Q) \geq \max (P)+d]$

$\llbracket \mathrm{TP} \rrbracket^{w_{0}}=1$ iff $\forall w\left[w \in A c c_{w_{0}} \rightarrow \max \{d: \operatorname{Height}(m)+d \leq \operatorname{Height}(j)\}=2^{\prime \prime}\right]$ 
Scope Ambiguities in Comparative Constructions

b. at least reading for Oda/Beck (differential takes wide scope) - 2 versions:
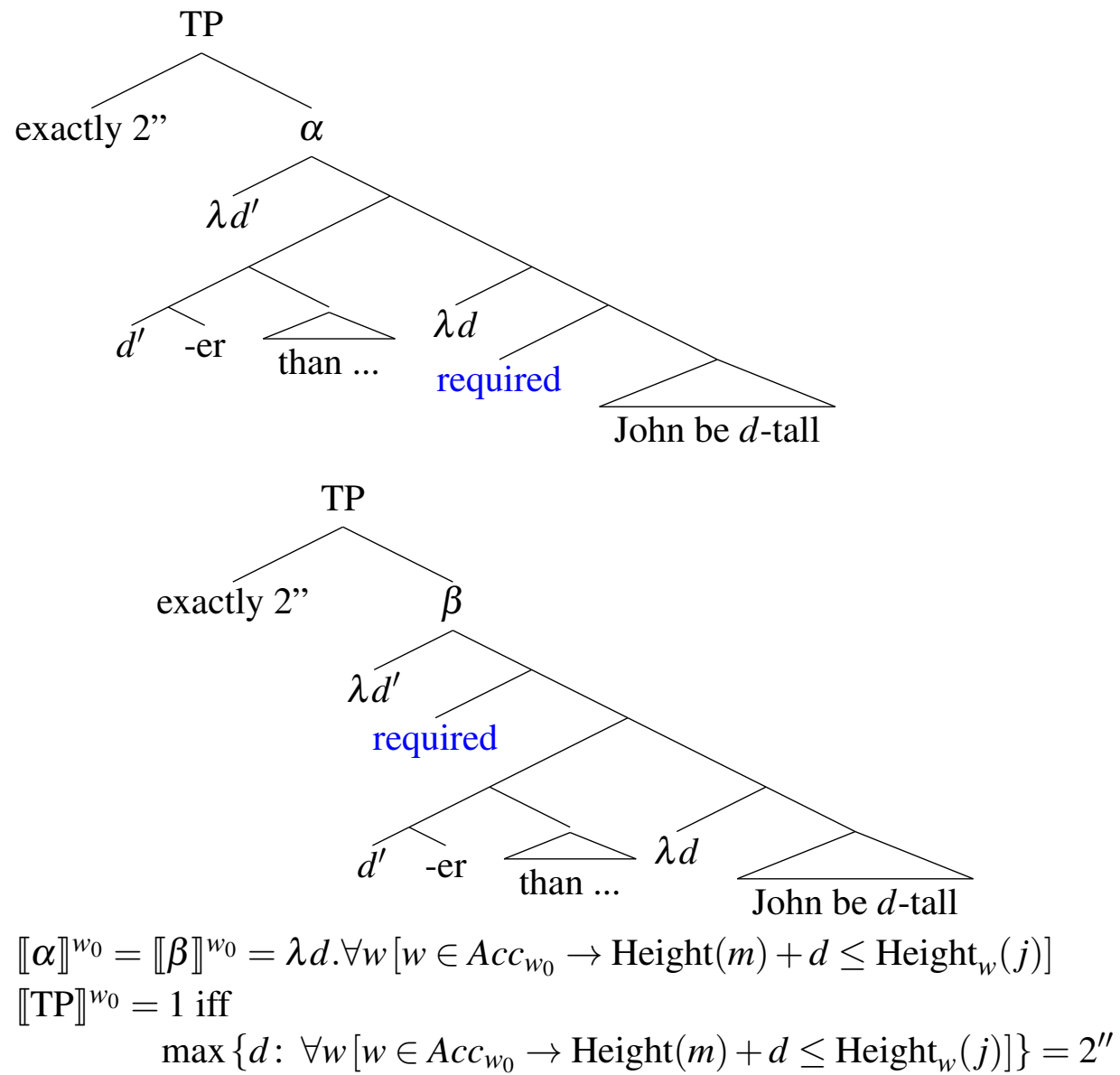

\subsection{An Argument for the exactly-scope Theory}

The goal of this paper is to draw predictions which distinguish the $e r$-scope theory from the exactly-scope theory and to see where the chips might fall. But first we would like to address what seems, at least at first sight, to be a strong argument for the exactly-scope theory. The argument comes from sentences which have an exactly differential but lack a comparative. When such sentences are embedded under certain modals, they seem to give rise to the same ambiguity that we have seen in (1). This argues that an exactly phrase is scopally active independently of the comparative and thus seems to tell us that O\&B's proposal has got to be correct.

To see this, consider the following sentence (based on Beck 2009: ex. 15):

(11) You are allowed to miss exactly 3 classes.

This sentence is ambiguous with two relevant meanings given by (14)a and b, an 
ambiguity which seems to motivate a treatment of exactly 3 as a quantifier over degrees, as in (12), with the two meanings derived from the two LFs in (13).

$\llbracket$ exactly $3 \rrbracket=\lambda P_{d t} \cdot[\max (P)=3]$

a. [allowed [[exactly 3$]_{1}\left[\left[\right.\right.$ you miss $t_{1}$ (many) classes $\left.\left.\left.]\right]\right]\right]$

b. [[exactly 3$]_{1}\left[\left[\right.\right.$ allowed [you miss $t_{1}$ (many) classes]]]]

a. $\exists w \in A c c: \max \{d$ : you miss $d$-many classes in $w\}=3$

It is permitted for you to miss exactly 3 classes

b. $\max \{d: \exists w \in A c c$ : you miss $d$-many classes in $w\}=3$

You are permitted to miss at most 3 classes

We conclude that an exactly phrase is indeed a quantifier over degrees which must be allowed to move by QR to its scope position. It seems to follow, then, that the structures assumed by the exactly-scope theory (in (8b)) must be available parses for the inverted scope reading of (1). Moreover, if quantifiers in object position cannot be interpreted in situ - as assumed by, e.g., Heim \& Kratzer 1998 - the structures proposed by the er-scope theory simply cannot be available parses.

This is transparent once we consider the denotation in (9) repeated below.

$$
\llbracket \text {-er } \rrbracket=\lambda d_{d} \cdot \lambda P_{d t} \cdot \lambda Q_{d t} \cdot[\max (Q) \geq \max (P)+d]
$$

If the semantics of the comparative in (9) is correct, an exactly differential (which has the denotation in (7)) is not the type of argument that -er is looking for. It can therefore not be interpreted in situ and QR must apply yielding the constituency assumed by the exactly-scope theory (unless some type flexibility is tolerated).

Furthermore, there is no obvious way to block QR of the exactly phrase from applying non-locally, yielding the structures for inverted scope proposed by the exactly-scope theory. And, given that an exactly phrase can QR non-locally (as indicated by the ambiguity of (11)), ad hoc stipulations that would block QR from applying in this particular case seem to be inappropriate. Therefore, at least as things stand right now, the er-scope theory doesn't seem to be a real competitor of the exactly-scope theory.

\subsection{A Way of Further Explicating Heim's Proposal}

In order to have two real competitors here, we need a version of (9) which would allow the complex -er phrase (the phrase that moves according to the er-scope theory) to be an interpretable constituent. The obvious thing to consider is modifying (9) so that the differential argument is not a degree but rather a generalized quantifier over degrees. With this in mind we would like to consider the following denotation: 
$\llbracket$-er $\rrbracket=\lambda M_{d t, t} \cdot \lambda P_{d t} \cdot \lambda Q_{d t} \cdot[M(\operatorname{Measure}(Q \backslash P)]$ where $Q \backslash P:=\{x: x \in Q \wedge x \notin P\}$; and Measure takes an interval and returns an interval of the same size with 0 as its left edge. ${ }^{2}$

Given (15), the comparative phrase "exactly 2 inches -er than 6 feet" can now receive a compositional interpretation, with the node $\alpha$ in (6) derived by functional application (assuming that "than 6 feet" denotes the interval $(0,6$ feet $]$ ):

$$
\begin{aligned}
& \llbracket \text { exactly } 2 \text { inches -er than } 6 \text { feet } \rrbracket=(\llbracket \text {-er } \rrbracket(\llbracket \text { exactly } 2 \text { inches } \rrbracket))(\llbracket \text { than } 6 \text { feet } \rrbracket)= \\
& \lambda Q_{d t} \cdot \max \left(\text { Measure }\left(Q \backslash\left(0,6^{\prime}\right]\right)\right)=2^{\prime \prime} \\
& \lambda Q_{d t} \cdot\left[\max (Q)-6^{\prime}\right]=2^{\prime \prime}
\end{aligned}
$$

O\&B's account of the ambiguity of (11) can be maintained and live happily with Heim's assumptions about constituency in our basic example in (1). Furthermore, Heim's constituency for the at least reading is predicted to be the only one available. There is no way to derive this reading by the structures assumed by O\&B. The trace left by such QR would have to be interpreted as a variable ranging over a quantifiertype, which would make this movement semantically vacuous (considerations that come from the de-dicto/de-re distinction do not arise for the quantifier exactly 2 inches which receives the same denotation across all worlds):

$\left[\left[\text { exactly } 2^{\prime \prime}\right]_{2}\left[\mathrm{John}\right.\right.$ is required $\left[\mathrm{t}_{<d t, t>, 2} \text {-er than } 6 \text { feet }\right]_{1}\left[\right.$ to be $\mathrm{t}_{d, 1}$ tall $\left.\left.]\right]\right]=$ $\llbracket$ exactly $2^{\prime \prime} \rrbracket\left(\lambda Q . \forall w\left[w \in A c c_{w_{0}} \rightarrow Q\left((\lambda d . \mathrm{J}\right.\right.\right.$ is d-tall $\left.\left.\left.) \backslash\left(0,6^{\prime}\right]\right)\right]\right)$ $\forall w\left[w \in A c c_{w_{0}} \rightarrow \max (P)-6^{\prime}=2^{\prime \prime}\right]$

We conclude that there is a version of the er-scope theory that makes it a real competitor of the exactly-scope theory. The question, now, is whether we can distinguish them on empirical grounds. Our answer is that we can - based on the central distinguishing property: under the exactly-scope theory, an inverted scope reading is derived by $\mathrm{QR}$ of the exactly differential, whereas under the er-scope theory, a larger constituent, the comparative phrase, undergoes $\mathrm{QR}$. The different predictions concern constituents that are contained in the larger constituent but not in the smaller one. To test these predictions, we will look at one such constituent, namely the than-clause, and we will probe for properties that are indicative of its position under the inverted scope reading.

\section{Offline Evidence Favoring the er-scope Theory}

According to the er-scope theory, the inverted scope interpretation can only result from a structure in which the than-clause is outside the scope of the relevant modal.

2 For example Measure when applied to the interval $(5,6]$ yields the interval $(0,1]$.

3 Whenever $\mathrm{P}$ is an interval of the form $(0, d]$. 
In order to see whether this prediction is correct we need to have methods for fixing the syntactic position of the than-clause. We will consider one such method, which stems from the de dicto/de re distinction. ${ }^{4}$

\subsection{De Re and De Dicto Readings}

Up to this point we have considered only one type of than-clause - "than 6 feet" - one whose interpretation is not dependent on the world of evaluation. The interpretation of such a than-clause does not provide any evidence pertaining to its syntactic position relative to intensional operators. In this sub-section we will consider than-clauses whose interpretation does depend on the world of evaluation and we will see that when they are evaluated in the world quantified over by the relevant intensional operator (when we get a de dicto interpretation for the thanclause) inverted scope for the relevant degree quantifier is unavailable.

Our test case involves an attempt to combine a de dicto interpreted than-clause with inverted scope for the relevant degree quantifier. It is, therefore, useful to have independent means to force inverted scope and de dicto so that we can see whether they can combine. We proceed one step at a time. In (18) we present an environment in which the inverted scope reading is the only available interpretation.

Inverted scope without controlling for de dicto/de re

Speaker A: What do I need to do in order to pay no taxes at all? Do I have to earn less than $20 \mathrm{~K}$ ?

Speaker B: Not quite. You are allowed to earn exactly $\$ 300$ more than that.

The relevant sentence is the one uttered by speaker B. Speaker B needs to be interpreted as asserting that $\$ 20,300$ is the maximum amount that you could earn and pay no taxes. This interpretation would result from wide scope of the relevant quantifier over the modal allowed resulting in an at most reading (under both theories). ${ }^{5,6}$

In (18), we used a simple than-clause, which does not allow us to probe the de dicto/de re distinction. In (19), we use a than-clause which can only receive a de dicto interpretation but in the context of a simple comparative that does not allow us to determine the scope of the relevant quantifier over degrees.

4 See Bhatt \& Pancheva 2004, example 54, for another argument related to extraposition of the than-clause.

5 We leave it to the reader to compute.

6 The reason why the utterance is restricted to inverse scope is that surface scope would be too weak in this context. For some reason, in this context B is understood to be providing a complete answer to the question under discussion and the surface scope interpretation is too weak to be a complete answer: all it says is that the rules do not exclude a salary of $\$ 20,300$. 
Scope Ambiguities in Comparative Constructions

(19) De dicto without controlling for surface-/inverted-scope

You are required to earn less than average.

Cf. You are required to earn less than last year's average

For reasons that are not entirely clear to us, the bare noun average can only receive the de dicto interpretation: the sentence is understood as reporting on a requirement that pertains to one's salary in relation to whatever turns out to be the average (rather than to a particular amount, e.g. last year's average).

Now we can ask whether we can combine the two. (20) suggests that we can't. ${ }^{7}$

A failed attempt to combine de dicto and inverted scope

Speaker A: What do I need to do in order to pay no taxes at all. Do I have to earn less than average?

Speaker B: \#Not quite. You are allowed to earn exactly $\$ 300$ more than average.

B's reply in (20) is infelicitous. We suggest that this stems from an attempt to combine the need for an inverted scope interpretation (the demand of context that we have seen in (18)) with the need for a de dicto interpretation of the than-clause (the demand of the bare noun that we have seen in (19)). This, as we said, is predicted by the er-scope theory but not by the exactly-scope theory.

\section{Predictions for Language Processing}

In this section we present experimental evidence from a sentence processing study that converges with what we have seen in Section 3. The experimental design we use is based on a paradigm explored in Hackl, Koster-Moeller \& Varvoutis submitted to study the effects of covert movement on real time sentence processing.

\subsection{Experimental Design}

The experimental design is based on two underlying assumptions: (a) that the linguistic parser, as a rule, postulates the simplest syntactic objects consistent with stimuli (an assumption with a long history - Bever 1970, Frazier \& Rayner 1982, Phillips 2003, etc.); and (b) that structures without QR are simpler than structures with QR (Anderson 2004, Fox 1995, Fox 2000, Tunstall 1998, etc.). Based on these

7 In (i)a and (i)b, below, we see that variations on (20), in which the than-clause can be interpreted de re or alternatively, surface scope is allowed, are acceptable.

i. A: What do I need to do in order to pay no taxes at all? Do I have to earn less than average?

a. B: Not quite. You are required to earn less than $\$ 300$ above average.

b. B: Not quite. You are allowed to earn exactly $\$ 300$ more than last year's average. 
assumptions QR would not be postulated in the parse of a sentence until the point in time at which the parser "realizes" that it is necessary. From that point on, one might expect to detect online consequences (e.g. a delay in reading time) of the shift to a less preferred parse.

Consider, from this perspective, how a left-to-right parser might process a sentence like (21), which involves the ambiguity we are interested in, but also involves an elided VP inside the than-clause whose antecedent is the matrix VP, (Antecedent Contained Ellipsis, henceforth ACE). ${ }^{8}$

(21) John was [Antecedent VP required to drive exactly $30 \mathrm{mph}$ faster $\left[\operatorname{than}_{2}\right.$ Mary was < Elided VP required to drive $t_{2}$ fast $>$ ]].

Resolving ACE requires a structural analysis of the sentence in which the elided VP is not part of the composition of its antecedent (e.g. Sag 1976, Williams 1977). In particular, it has been argued to require $\mathrm{QR}$ of the comparative phrase, which hosts the than-clause, placing it above the matrix VP, effectively undoing antecedent containment, (22). ${ }^{9}$

(22) [exactly $30 \mathrm{mph}$-er [than 2 Mary was < required to drive $t_{2}$ fast $\left.>\right]_{1}$ [John was [required to drive $t_{1}$-fast]].

Given that ACE resolution requires $\mathrm{QR}$, we might expect online consequences associated with the ACE site that are indicative of a shift to a parse that involves QR. This expectation should hold, however, only if there is no pressure for the shift to occur earlier on.

Here is where the two theories discussed in sections 1-3 differ. Under the erscope theory such pressure would exist if the context somehow indicated that the sentence is to receive an at least reading whereas under the exactly-scope theory it wouldn't. Under the er-scope theory the at least reading requires a parse in which the -er phrase moves over the modal, and the need for such a parse would be evident the moment the differential exactly $30 \mathrm{mph}$ is parsed. Since this instance of QR carries along the than-clause it would be sufficient to accommodate ACE, removing the need for reanalysis when the actual ACE site is reached.

In other words, under the er-scope theory we would expect that an upstream occurrence of an exactly differential can facilitate the resolution of a nonlocal ACE site downstream, since the $\mathrm{QR}$ related portion of the work involved in ACE resolution has been completed already before the ACE site is reached. Under the exactly-scope theory, however, the need to derive an at least reading would motivate non-local

8 We assume, as is standardly done, that the than-clause contains an operator in its left periphery that abstracts over the degree position.

9 See e.g. Heim 2000. 
scope for just the exactly differential, since the at least reading can be generated with the comparative phrase inside the scope of require. Parsing the differential would, then, not yield any indication to the parser to shift to a parse that could accommodate a non-local ACE site downstream. Only when the parser reaches the ACE site itself, will the need for non-local movement of the comparative phrase become apparent. Thus, under the exactly-scope theory, we do expect online consequences associated with the ACE site that are attributable to $\mathrm{QR}$ and we do not expect that an exactly differential could facilitate the resolution of a non-local ACE site inside the than-clause.

To test these predictions empirically, two ingredients need to be in place: i) we need a way of forcing an at least reading for sentences with exactly differentials and ii) we need a baseline that allows us to assess the processing costs incurred by a non-local ACE site in a than-clause in the absence of an upstream trigger for non-local QR. Our strategy to ensure an at least reading is to construct sentences, such as the one in (23), which, given world knowledge, make an exactly reading extremely implausible.

(23) In order to become the all-time champion, John was required to win exactly 3 more races than Bill.

The sentence in (23) is, in principle, ambiguous between an exactly and an at least reading. However, given what we know about sports records, the exactly reading is highly implausible: it would be rather peculiar if John's accomplishments were to somehow be diminished (so that he would no longer be the all-time champion) if he won a few additional races.

Our strategy to generate a baseline that allows us to assess the processing costs of non-local ACE in the absence of an upstream trigger for non-local QR is to compare processing costs of sentences with what we will call at least differentials (differentials which do not give rise to Heim-style scope ambiguities) across two levels of ACE (local and non-local), as illustrated in (24): ${ }^{10}$

a. John was required to win a few more races than Bill did <win d-many races $>$.

b. John was required to win a few more races than Bill was <required to win d-many races $>$.

The sentences in (24) differ from the previous sentences we considered (e.g. (23)) in that the differential argument of the comparative is realized by a few rather than by an exactly phrase. These sentences are not ambiguous. They have only one

10 An alternative baseline would result from omitting the differential altogether. Our choice was made for technical reasons: to keep sentences at equal length with the relevant words correctly aligned. 
interpretation which is close to the at least reading of Heim's sentences (as far as consistency with world knowledge is considered). That is, neither (24a) nor (24b) seem to have an exactly entailment. Since nothing would preclude interpreting the differential locally in the scope of require, we conclude that a few is a differential that gives rise to at least truth-conditions irrespective of whether it is integrated above or below the modal operator. ${ }^{11}$

Because of its scopal inertness with regard to the modal operator, parsing a few will not constitute evidence for the parser to shift to a parse with non-local QR of the comparative phrase. Evidence to that effect arises only at the ACE site itself. This allows us, then, to measure processing costs of non-local QR at the ACE site in the absence of an upstream trigger for non-local QR.

The way we propose to measure these costs is to compare non-local ACE against local ACE. That is, both (24a) and (24b) involve an ACE site in the than-clause. The ACE sites are, however, marked by different auxiliaries, which govern differently sized ACE sites. Specifically, when the ACE site is marked by did, as in (24a), the antecedent of the elided constituent is the local VP projected from win and we are dealing with a case of local ACE. When the ACE site is marked by was, as in (24b), the antecedent is the matrix VP projected from require. In this case, we are dealing with non-local ACE. Thus, comparing the processing costs associated with the markers for local and non-local ACE provides us with a measure of how difficult it is to resolve non-local ACE in the absence of an upstream trigger for non-local QR. This measure can, thus, serve as a baseline against which we can compare our test case: non-local ACE when there is an upstream exactly differential.

Putting these considerations together we get an experimental paradigm that crosses two factors (Differential-Type and ACE-Size) as exemplified in (25).

(25) In order to become the all-time champion, the American athlete was required to win...

a. exactly 3 more matches than the British athlete did

(local ACE)

b. exactly 3 more matches than the British athlete was

(non-local ACE)

c. a few more matches than the British athlete did

(local ACE)

d. a few more matches than the British athlete was

(non-local ACE)

... and so practiced arduously for several months.

11 See Heim 2000 for a general discussion of when degree quantifiers can give rise to scope interactions that are detectable in terms of their truth-conditions. Note that a few can give rise to an implicature ("not a lot") and that this implicature can (perhaps must) be interpreted above require. However, this implicature is computed in the same way under any possible scope of the degree quantifiers we are studying here. 
Scope Ambiguities in Comparative Constructions

\subsection{Predictions}

To recap, our design embeds target sentences, which involve either an exactly differential or an at least differential and either local or non-local ACE, in contexts that strongly favor at least readings. For sentences with a few, $(25 \mathrm{c}, \mathrm{d})$, generating an at least reading does not depend on the scope of the differential. Thus, the comparison of processing costs at or after the ACE site will give us a baseline against which we can measure potential effects of an upstream exactly differential on processing non-local ACE. We expect our baseline conditions to display an increase in processing cost for non-local ACE (in comparison to local ACE) since resolving non-local ACE involves reanalysis in favor of non-local QR. ${ }^{12}$

For the sentences with an exactly differential our expectation depends on the assumptions we make about how at least readings are generated. Under the erscope theory generating the at least reading requires non-local scope of the entire comparative phrase. Encountering the exactly differential will, therefore, trigger reanalysis of the sort that can accommodate non-local ACE. Thus, when the parser reaches the non-local ACE site no further reanalysis is required and we expect less of an increase in processing cost when comparing local to non-local ACE. Under the exactly-scope theory, by contrast, we expect no facilitation effect since under this theory evidence for the need to move the comparative phrase above the modal is obtained only at the point where the non-local ACE site is identified.

\subsection{Methods and Materials}

We constructed 44 sentence templates following the sample paradigm in (25). Sentences begin with a context-providing clause favoring the at least reading with length varying between 4 and 16 words, identical for all sentences in a given template. Each sentence in a template employed a comparative with a differential headed by either exactly or a few. The comparative was constructed with a than-clause hosting either local or non-local ACE. Sentences were constructed so that the ellipsis marker did was naturally interpreted to involve local ACE, while the ellipsis marker was was naturally interpreted to involve non-local ACE. Modifiers were inserted between the differential and the ACE site to create a large enough separation between the two regions of interest so that possible spill-over effects due to a differential would not interfere with the processing of material in the second area of interest (the differential and the ellipsis site were separated by exactly seven words in all our items). Following the ellipsis site, conjunctions headed by and, which varied in length between 5 and 14 words, were employed to provide a spill-over region for

12 There might also be a cost involved in the retrieval of a larger, more complex antecedent, but that should not affect our expectations in a qualitative way. 
detecting possible processing difficulty associated with ACE. These conjunctions were identical for all sentences of a given template.

Target items were counterbalanced across four lists using a Latin-square design and combined with 28 sentences of an unrelated experiment as well as 81 filler sentences of various types resulting in a total of 153 experimental sentences. Nontarget items included sentences that were similar to the target items in structure (employing degree clauses, comparatives or elided material) and in length.

Thirty seven participants recruited by and run through MIT's Behavioral Research Lab, were tested on Macintosh computers running the Linger software developed by Doug Rohde. All subjects were native speakers of English and received $\$ 10.00$ cash compensation for their participation. Each trial began with two series of dashes on two separate lines, the first marking the length of the contextual clause and the second marking the length of the remainder of the sentence. Participants press the space bar to reveal the next word of the sentence. Each press of the space bar reveals a new word while the previous word is again replaced by dashes. Apart from the line separator, there is no distinction between the contextual clause and the remainder of the sentence. The amount of time a participant spends reading each word is recorded (RT). After the final word of each sentence, a yes/no comprehension question appears, asking about information contained in the sentence. Participants respond by pressing keys marked on the keyboard. If an incorrect answer is given, the message "Oops! Wrong answer!" appears on the screen to encourage participants to read more carefully. Participants are instructed to read sentences at a natural rate and to be sure that they understand what they read. They are also instructed to answer the questions as accurately as possible. Completion of the experiment took between 45 and 60 minutes on average.

\subsection{Results}

Questions across the full experiment (targets and fillers) were answered correctly $89.7 \%$ of the time across participants; questions for experimental items were answered correctly on $88.4 \%$ of trials. A two-factor ANOVA crossing differential (exactly versus a few) with ACE (local, non-local) on the correctness-rates revealed no significant differences (rates were $90.1 \%, 86.9 \%, 87.9 \%$ and $88.1 \%$ on the four conditions respectively). We excluded participants who did not answer more than $75 \%$ of the total items (targets and fillers) or more than $75 \%$ of the target items correctly. Only one participant was excluded based on these criteria. For all remaining participants, data from sentences with incorrectly answered questions was removed. Below we report residual reading times (RRTs). However, we observe the same effects in the raw reading times. RRTs were calculated based on a regression equation predicting reading time from word length using all words from all exper- 


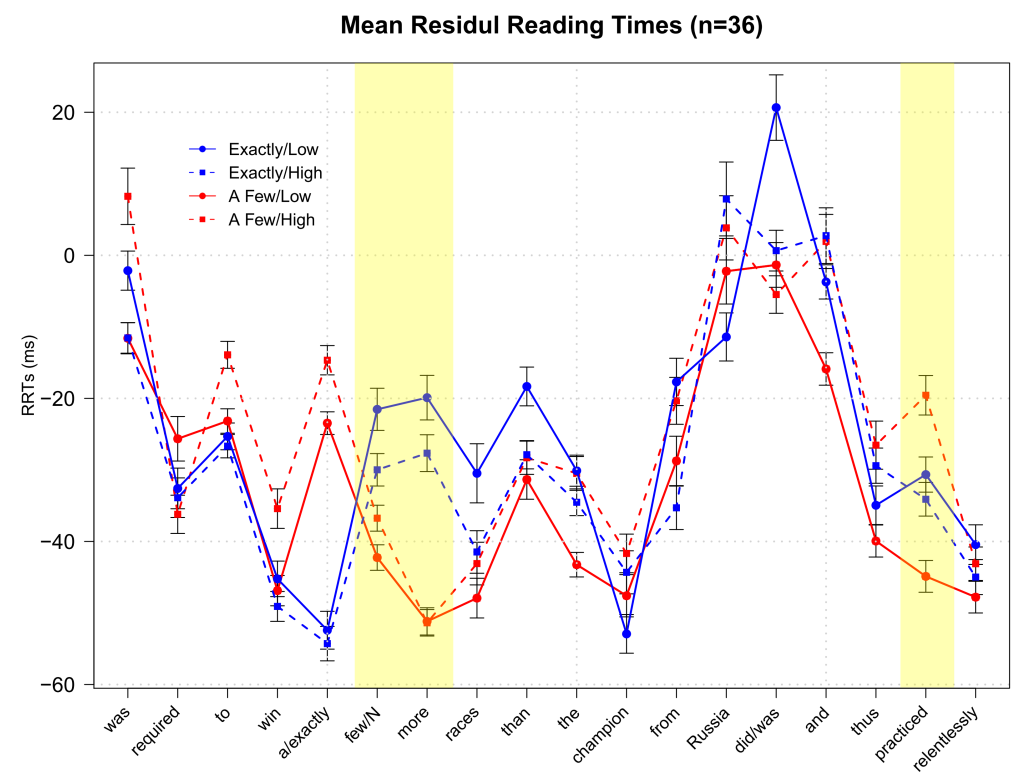

Figure 1 Mean residual reading times.

imental items (except the last word in a sentence). For each participant, residual reading times beyond two standard deviations from the mean for a given condition and position were excluded from analyses. Averaged word by word RRTs in the regions of interest are shown in Figure 1.

The two regions of interest are marked in yellow in Figure 1. Visual inspection of the reading times at the differential site (a/exactly in Figure 1) suggests that on the first word of the differential, RRTs for exactly are below RRTs for $a,{ }^{13}$ while on the second word of the differential, as well as on the comparative more, the pattern is reversed. A contrast coded and centered linear mixed effects model with Differential and ACE-size as factors reveals a main effect of Differential on a/exactly, few/ $\mathrm{N}$, and more ( $\mathrm{pMCMC} ; \mathrm{p}=0.001, \mathrm{p}=0.0016, \mathrm{p}=0.001$, respectively). No comparably significant effects were found until the site of the ellipsis marker (did/was in Figure 1) within the matrix clause.

The second region where we see an effect of our factors is on the third word after the ellipsis site, P3 (practiced in Figure 1). We see that (i) RRTs for the [exactly/was] condition are lower than RRTs for the [a few/was] condition; and (ii) RRTs for the

13 Note that word length is factored in, though the linear model used to estimate RRTs by word length underestimates predicted RRTs of words of length 1 such as $a$. Indeed, raw RTs do not show a difference between exactly and $a$, yet there is a significant difference for the next two words showing that exactly differentials are slower than a few. 


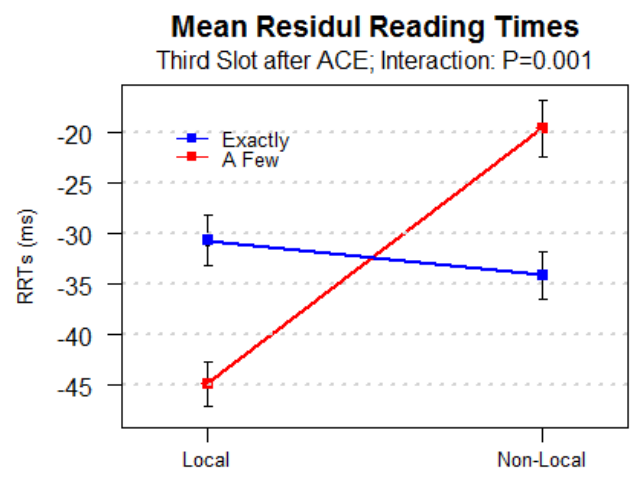

Figure 2 Interaction associated with ACE site.

[exactly/did] condition are higher than RRTs for the $[$ a few/did] condition. This can be seen more clearly in Figure 2 where we present average RRTs at P3.

We again fit a contrast coded and centered linear mixed effects model to the data and find a Differential by ACE-size interaction on P3 (pMCMC; p=0.002), and a main effect for ellipsis size $(\mathrm{p}=0.023)$. Additionally, pooling RRTs for the first 3 words after the ellipsis site (and, thus, practiced in Figure 1) also reveals the same Differential by ACE-size interaction ( $\mathrm{MMCMC} ; \mathrm{p}=0.019$ ), and a main effect for ellipsis size $(\mathrm{p}=0.028)$. No other effects were detected within this region.

\subsection{Discussion}

The results at the differential site indicate that an exactly differential incurs increased processing time compared with an a few differential on the two first words following the differential. This could simply be due to low level differences between $a$ few and exactly such as lexical frequency, but it could also be indicative of exactly triggering QR to yield a wide scope reading. Our data do not allow us to distinguish between these two interpretations. Furthermore, even if it could be shown to be linked to QR, it would not distinguish between the er-scope and the exactly-scope theories, since both identify the exactly differential as a trigger for reanalysis.

The results associated with the ACE site, however, do distinguish between the two theories. The er-scope theory predicts facilitation of non-local ACE while the exactly-scope theory does not. Our findings support the er-scope theory. Specifically, the interaction at P3 (shown in Figure 1), and throughout the three word region after the auxiliary, is the product of a marked increase in RRTs for non-local ACE when 
the differential is a few that is missing when the differential is exactly. This indicates that an upstream exactly differential facilitates the resolution of a non-local ACE site downstream in our sentences, just like the er-scope theory predicts. The exactlyscope theory, by contrast, does not make this prediction.

Note that there is an aspect of our results that neither theory predicted, namely the fact that the processing costs associated with the local ACE site are higher when the upstream differential was an exactly phrase compared to when it was a few. Under the er-scope theory, this could be seen as a consequence of the preference principle in (26) - inspired by Hardt \& Romero 2004 - according to which the scope of an expression that hosts an ACE should match the size of elided constituent. ${ }^{14}$

(26) Scope-matching preference principle: The scope of a phrase hosting an ACE site is the lowest interpretable position that allows ACE resolution.

Sentences that employ an exactly differential in combination with the ACE marker did, the [exactly, did] condition, violate (26), if the er-scope theory is correct. Under this theory, the at least reading requires non-local scope of the comparative phrase but this is not the lowest interpretable position that would allow ACE resolution, as required by the preference principle. The $[a \mathrm{few}, \mathrm{did}]$ condition, by contrast, involves no mismatch in scope and ACE size since the at least reading can be generated when the comparative phrase is interpreted just above the local VP, which is the antecedent of the ACE site marked by did. Under the exactly-scope theory, this line of explanation is not available.

Returning to our main point, our results show that the choice of differential upstream has interactive rather than additive consequences for the processing costs associated with downstream ACE sites. Moreover, the interaction we observe indicates facilitation of non-local ACE resolution by an upstream exactly differential since there is no increase in processing cost for non-local ACE under that condition. This is expected by the er-scope theory but not by the exactly-scope theory.

\section{Summary}

In this paper we asked whether differentials take scope independently of the comparative. We investigated this question with both off-line data and in the arena of real-time sentence processing, with converging evidence for a negative answer. The er-scope theory, which is based on an analysis of the comparative as a quantifier that itself takes a quantifier as an argument, was presented as an explanation for both the

14 The principle is weaker than Hardt and Romero's original claim and intended as a parsing principle rather than as a grammatical principle that can be sidestepped under certain discourse conditions. See Takahashi 2008 for arguments against Hardt and Romero's grammatical principle. See also Frazier \& Clifton 2005. 
offline evidence and the processing results. Under such a theory, an exactly phrase, which is a quantifier over degrees, is scopally active when it is base generated in a degree argument position. But when it is the differential argument of -er, it is scopally inert and its contribution to interpretation is parasitic on the scope of -er.

The results of our experiment also support the claim that $\mathrm{QR}$ has detectable effects on real time sentence processing. More specifically, they provide further corroboration for the Hackl et al. submitted claim about the way potential triggers for QR interact, namely that an upstream trigger will facilitate the processing of downstream material that would have otherwise been the sole trigger for QR.

\section{References}

Anderson, Catherine. 2004. The structure and real-time comprehension of quantifier scope ambiguity: Northwestern University dissertation.

Beck, Sigrid. 2009. DegP scope revisited. Ms., Universität Tübingen .

Bever, Thomas G. 1970. The cognitive basis for linguistic structures. In J.R. Hayes (ed.), Cognition and the Development of Language, 279-362. Wiley, New York.

Bhatt, Rajesh \& Roumyana Pancheva. 2004. Late merger of degree clauses. Linguistic Inquiry 35(1). 1-45.

Fox, Danny. 1995. Economy and scope. Natural Language Semantics 3(3). 283-341.

Fox, Danny. 2000. Economy and Semantic Interpretation. Cambridge, Mass.: MIT Press: 727-796.

Frazier, Lyn \& Charles Jr Clifton. 2005. The syntax-discourse divide: Processing ellipsis. Syntax 8. 121-174.

Frazier, Lyn \& Keith Rayner. 1982. Making and correcting errors during sentence comprehension: Eye movements in the analysis of structurally ambiguous sentences. Cognitive Psychology 14. 178-210.

Hackl, Martin, Jorie Koster-Moeller \& Jason Varvoutis. submitted. Quantification and ACD: Evidence from real time sentence processing.

Hardt, Daniel \& Maribel Romero. 2004. Ellipsis and the structure of discourse. Journal of Semantics 21(4). 375-414.

Heim, Irene. 2000. Degree operators and scope. Proceedings of SALT X, 40-64.

Heim, Irene \& Angelika Kratzer. 1998. Semantics in Generative Grammar. Wiley Blackwell.

Kennedy, Christopher. 1997. Projecting the adjective: The syntax and semantics of gradability and comparison: University of California Santa Cruz dissertation.

Oda, Toshiko. 2008. Degree constructions in Japanese: University of Connecticut dissertation.

Phillips, Collins. 2003. Parsing: Psycholinguistic aspects. In International Encyclopedia of Linguistics, 2nd. edn., Oxford University Press. 
Scope Ambiguities in Comparative Constructions

Sag, Ivan A. 1976. Deletion and logical form: MIT dissertation.

von Stechow, Arnim. 1984. Comparing semantic theories of comparison. Journal of Semantics 3. 1-92.

Takahashi, Shoichi. 2008. Variable binding in temporal adverbial clauses: Evidence from ellipsis. Proceedings of WCCFL 26.

Tunstall, Susanne L. 1998. The interpretation of quantifiers: Semantics and processing: UMass dissertation.

Williams, Edwin. 1977. Discourse and logical form. Linguistic Inquiry 8. 101-139.

Micha Breakstone

micha.breakstone@gmail.com

http://web.mit.edu/michab/www/index.html

Danny Fox

fox@mit.edu
Alexandre Cremers

alexandre.cremers@gmail.com

Martin Hackl

hackl@mit.edu 